\title{
Silicon Microspheres for Wavelength Division Multiplexing in the THz Band
}

\author{
Ali Serpengüzel* and Onur Akatlar \\ Koç University, Microphotonics Research Laboratory, Physics Department, \\ Rumeli Feneri Yolu, Sariyer, Istanbul 34450 Turkey
}

\begin{abstract}
Microspheres possess high quality factor morphology-dependent resonances, i.e., whispering gallery modes. Additionally, silicon is proving itself to be an excellent optical material. We have studied the feasibility of silicon microspheres as a optical filters at $\mathrm{THz}$ communication frequencies. The silicon microsphere has a radius of $500 \mu \mathrm{m}$ and a refractive index of 3.4. Elastic scattering spectra are calculated for TE and TM polarizations for wavelengths between $90 \mu \mathrm{m}$ and $100 \mu \mathrm{m}$ in the THz communication band. The high quality factor morphology dependent resonances are found to have a repetitive mode spacing of $1.2 \mu \mathrm{m}$.
\end{abstract}

Keywords: channel dropping, detector, frequency selective, microsphere, morphology dependent resonance, light coupling, optical fiber coupler, whispering gallery mode.

\section{INTRODUCTION}

Dielectric microspheres have found various photonic applications in the ultraviolet (UV), visible and near-infrared (IR) communication bands [1]. Morphology dependent resonances (MDR's) or alternatively whispering gallery modes (WGM's) of dielectric microspheres provide the necessary optical feedback for applications in spectroscopy, laser science, and optical communications. Among these applications, we can find novel microlasers, optical couplers, and optical filters. Low threshold lasing from rare earth doped silica microspheres [2], polymer microsphere lasers [3] and Raman lasers have been demonstrated. Strain tunable microsphere oscillators [4], adddrop filters [5], and thermooptical switching [6] have been realized for the frequency control in optical communications. Microsphere resonators are uniquely applicable in compact optoelectronic devices in wavelength division multiplexing (WDM) applications $\left.{ }^{7}\right]$. WDM has the advantage of increasing the bandwidth of any communication network. In WDM, the final optical to electronic conversion needs an all-optical packet-switching layer, which consists of all optical gates, interferometers, semiconductor optical amplifiers, resonant cavity enhanced (RCE) photodetectors, optical random access memory (RAM) elements, and channel dropping filters. In these planar lightwave circuits, microspheres can be used as compact channel dropping filters. Recently, we have observed elastic light scattering in silicon microspheres in the near-IR $\left[{ }^{8}\right]$.In this paper, we are investigating the far IR communication applications of silicon microspheres. Although, there is atmospheric absorption in some ranges of the $\mathrm{THz}$ band, this communication band can be used in space based communication applications. The system can easily be red or blue shifted by adjusting the size of the silicon microsphere and the operation frequency.

\section{MORPHOLOGY DEPENDENT RESONANCES}

The MDR's of the microsphere can be understood using geometrical optics. As the light propagates around the microsphere, it is confined by almost total internal reflection (TIR) [ $\left.{ }^{9}\right]$. After circumnavigating the microsphere, the

\footnotetext{
* Correspondence should be addressed to Ali Serpengüzel, aserpenguzel@ku.edu.tr; phone: + 90 (212) 338-1312; fax: + 90 (212) 3381547, http://home.ku.edu.tr/ aserpenguzel, http://microphotonics.ku.edu.tr, Koç University, Microphotonics Research Laboratory, Physics Department, Rumeli Feneri Yolu, Sariyer, Istanbul 34450 Turkey. The authors are with Koç University, Microphotonics Research Laboratory, Physics Department, Rumeli Feneri Yolu, Sariyer, Istanbul 34450 Turkey.
} 
light wave returns to the original starting point in phase to interfere constructively with itself. This constructive interference can occur only at certain discrete MDR wavelengths $\left[{ }^{10}\right]$. Each MDR is characterized by a mode number (n) and a mode order (1). Physically, (n) indicates the number of nodes in the internal intensity distribution as the polar angle is varied from $0^{\circ}$ to $180^{\circ}$. The mode order (l) indicates the number of nodes in the internal intensity distribution in the radial direction. For each set of mode number (n) and mode order (l), there is a transverse electric (TE) and transverse magnetic (TM) MDR. The mode spacing $\Delta \lambda=\left(\lambda^{2} \arctan \left(\mathrm{m}^{2}-1\right)^{1 / 2}\right) /\left(2 \pi \mathrm{a}\left(\mathrm{m}^{2}-1\right)^{1 / 2}\right)$, i.e. wavelength difference between consecutive mode numbers $n$ in the same mode order $l$, gives information about the size of the microsphere $\left[{ }^{11}\right]$. The $\mathrm{Q}$-factor of a MDR, $(\mathrm{Q}=\lambda / \delta \lambda)$, gives information about the lifetime and linewidth $(\delta \lambda)$ of the resonance $\left[{ }^{12}\right]$. For a given microsphere, the MDR occurs at specific value of the size parameter, $\mathrm{x}_{\mathrm{n}, \mathrm{l}}$, which is given by $2 \pi \mathrm{a} / \lambda_{\mathrm{n}, \mathrm{l}}$, where $\lambda_{\mathrm{n}, 1}$ is the light wavelength in vacuum and a is the radius of the microsphere. These MDR's have been verified experimentally at optical wavelengths with micrometer sized spheres.

\section{SILICON MICROSPHERES}

Silicon has been the material of choice for the electronics industry for more than half-a-century. It is a relatively inexpensive, plentiful, and well understood material for producing electronic devices $\left[{ }^{13}\right]$. Additionally, the need for low cost photonic devices has stimulated a significant amount of research in silicon photonics $\left[{ }^{14}\right]$. Although silicon photonics is less well developed as compared to III-V technologies; it is poised to make a serious impact on the telecommunications industry, as well as in many other photonic applications. Recent studies show that the silicon will be widely used, as in electronics, in the photonics industry $\left[{ }^{15}\right]$. Silicon microspheres with their morphology dependent resonances (MDR's) can also be used in $\mathrm{THz}$ communication applications, where silicon is transparent. This makes silicon the material of choice for micro-electro-photonic-integration (MEPI) and future electro-photonic integrated circuits (EPICs).

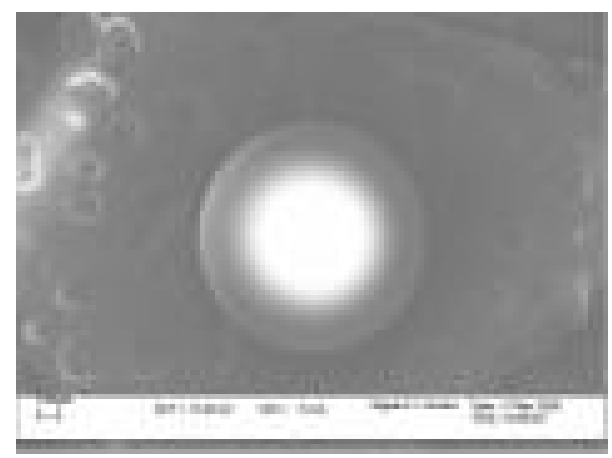

Fig. 2. SEM of the silicon microsphere.

The high purity single crystal silicon microspheres are produced by the solidification of molten silicon melt $\left[{ }^{16}\right]$. The silicon microspheres are then mechanically lapped and polished to make mirror-like finish with no surface defects and damages $\left[{ }^{17}\right]$. In order to investigate the sphericity of our microresonators, we have obtained SEM images of the microspheres $\left[{ }^{18}\right]$. Fig. 1 shows a scanning electron microgram (SEM) of the silicon microsphere.

\section{ELASTIC SCATTERING CALCULATIONS}

We have investigated the feasibility of $\mathrm{THz}$ communication filters using silicon microspheres. Elastic scattering calculations are performed between the wavelengths of $90 \mu \mathrm{m}$ to $100 \mu \mathrm{m} .0^{\circ}$ (forward), $90^{\circ}$, and $180^{\circ}$ (backward) elastic scattering spectra from a silicon microsphere of radius $500 \mu \mathrm{m}$ are calculated. Figure 2 shows the geometry of the elastic scattering calculations. 


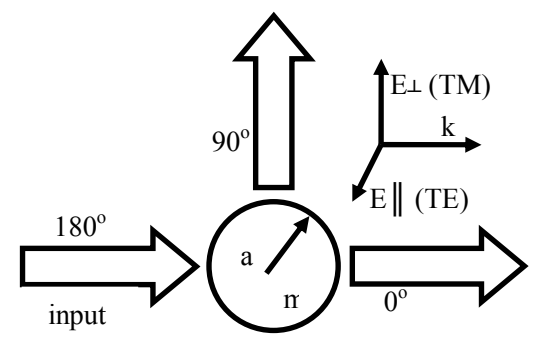

Fig. 2. Geometry of the elastic scattering calculations.

Figure 3 shows the results of the calculations for backward $\left(180^{\circ}\right)$ scattering from a microsphere of radius a $=500$ $\mu \mathrm{m}$. The mode spacing, i.e., the separation between the adjacent MDRs of the same mode number (l) with consecutive mode numbers (n) is estimated and calculated to be $1.2 \mu \mathrm{m}$. Note that because of symmetry, the TE and $\mathrm{TM}$ components give the same results for $180^{\circ}$ scattering. There is no background in the backward direction and the signal to noise is approximately 100 .

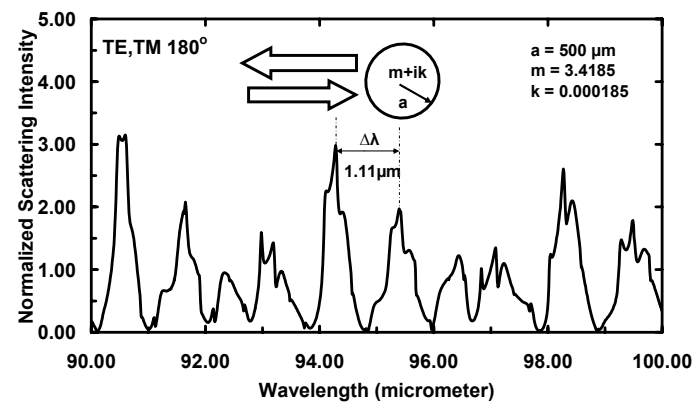

Fig $3.180^{\circ}$ normalized scattering intensity from a silicon microsphere.

Note that because of symmetry, the TE and TM components give the same results for $0^{\circ}$ scattering as well. There is a background in the forward direction and the signal to noise is approximately 3 . For the calculations for $90^{\circ}$ scattering from a microsphere of radius $\mathrm{a}=500 \mu \mathrm{m}$ for TE and TM polarizations, the spacing between the adjacent MDR peaks is $2.4 \mu \mathrm{m}$. This mode spacing value is twice the calculated mode spacing of $1.2 \mu \mathrm{m}$. That is due to the fact that, only even mode number resonances are observed at $90^{\circ}$ scattering [14]. There is no background in the $90^{\circ}$ direction and the signal to noise is approximately 100 .

\section{CONCLUSIONS}

We have calculated the elastic scattering spectra of silicon microspheres in the THz band. The mode spacing $\Delta \lambda$, i.e. wavelength difference between consecutive mode numbers (n) in the same mode order (1), is estimated and calculated to be $1.2 \mu \mathrm{m}$. The $90^{\circ}$ elastic scattering occurs only for even mode number MDRs, as a consequence, the mode spacing is two times bigger than any other angle The linewidths $\delta \lambda$ of the highest mode order MDR's are measured to be $1 \mu \mathrm{m}$, which results in a quality factor $(\mathrm{Q}=\lambda / \delta \lambda)$ of approximately $10^{2}$. With the proper system design, it would be possible to use silicon microspheres for space and earth based communication applications. The silicon microsphere shows promise as a building block for future microoptoelectronic integration.

\section{ACKNOWLEDGMENTS}

We would like to acknowledge the partial support of this research by the European Commission Grant No: FP6-IST511616: PHOREMOST and FP6-IST-003887 NEMO.

\section{REFERENCES}


1. S. M. Spillane, T. J. Kippenberg, and K. J. Vahala, "Ultralow-threshold Raman laser using a spherical dielectric microcavity", Nature, vol. 415, pp. 621-623, Feb. 2002.

2. V. Lefèvre-Seguin and S. Haroche, "Towards cavity-QED experiments with silica microspheres", Mat. Sci. Eng., vol. B48 pp. 53-58, 1997.

3. M. Kuwata-Gonokami and K. Takeda, "Polymer whispering gallery mode lasers", Opt. Mat., vol. 9, pp. 12-17, Jan. 1998.

4. V. S. Ilchenko, P. S. Volikov, V. L. Velichansky, F. Treussart, V. Lefèvre-Seguin, J.-M. Raimond, and S. Haroche, "Strain-tunable high-Q optical microsphere resonator", Optics Comm., vol.145, pp. 86-90, Jan. 1998.

5. M. Cai, G. Hunziker, and K. J. Vahala, "Fiber-optic add-drop device based on a silica microsphere- whispering gallery mode system," IEEE Photon. Technol. Lett., vol. 6, no. 6, pp. 686-687, June 1999.

6. H. C. Tapalian, J.-P. Laine, and P. A. Lane, "Thermooptical switches using coated microsphere resonators", IEEE Photon. Technol. Lett., vol. 14, no. 8, pp. 1118-1120, Aug. 2002.

7. A. Serpengüzel, S. Arnold, G. Griffel, and J. A. Lock, "Enhanced coupling to microsphere resonances with optical fibers," J. Opt. Soc. Am. B., vol. 14, no. 4, pp. 790-795, Apr. 1997.

8. Y.O. Yilmaz, A. Demir, A. Kurt, and A. Serpengüzel, "Optical Channel Dropping with a Silicon Microsphere," IEEE Photon. Technol. Lett., 17, 1662-1664 (2005).

9. M. Pelton and Y. Yamamoto, "Ultralow threshold laser using a single quantum dot and a microsphere cavity", Phys. Rev. A, vol. 59, no. 3, pp. 2418-2421, March 1999.

10. B. R. Johnson, "Morphology-dependent resonances of a dielectric sphere on a conducting plane", J. Opt. Soc. Am. A, vol. 11, no. 7, pp. 2055-2064, July 1994.

10. P. Barber, and S. C. Hill, Light Scattering by Particles: Computational Methods. Singapore: World Scientific, 1990.

11. M. L. Gorodetsky, A. A. Savchenkov, and V. S. Ilchenko, "Ultimate Q of optical microsphere resonators," Optics Letters, vol. 21, pp. 453-455, Apr. 1996.

13. L. Pavesi, "Will silicon be the photonic material of the next millennium?," J. Phys. Condensed Matter 15, pp. 1169-1196, 2003.

14. L. Pavesi and D. J. Lockwood, Silicon Photonics, New York: Springer-Verlag, 2004.

15. O. Boyraz, B. Jalali, "Demonstration of a silicon Raman laser," Optics Express, vol. 12, pp. 5269-5273, Oct. 2004.

16. W. R. McKee, "Development of the Spherical Silicon Solar Cell," IEEE Trans.Compon. Hybr. Manuf. Tech., vol. CHMT-5, no.4, pp. 336-341, 1982.

17. N. Takeda, "Spherical Silicon 1mm Device and its Clustering," Int. Symp. Advan. Pack. Mater., pp.86-91, 2001.

18. S. Lacey, H. Wang, D. H. Foster, and J. U. Nöckel, "Directional Tunneling Escape from Nearly Spherical Optical Resonators," Phys. Rev. Lett., vol. 91, no. 3, pp. 33902-33905, 2003. 